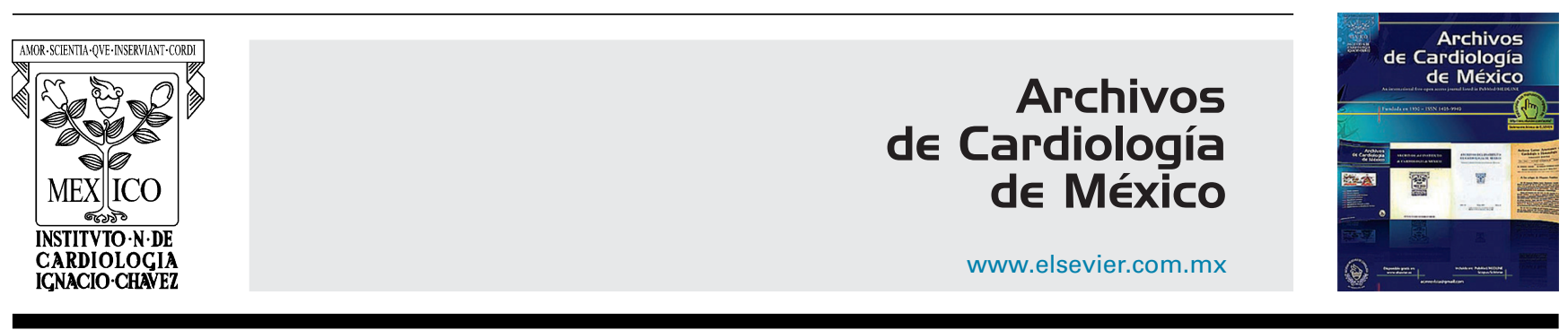

INVESTIGACIÓN CLÍNICA

\title{
Cirugía de revascularización de miocardio usando arteria mamaria interna bilateral. Resultados a corto plazo
}

\author{
Josías Caleb Ríos Ortega ${ }^{a, *}$, Paul Castañeda Castillo ${ }^{\mathrm{b}}$, Luisa Talledo Paredes ${ }^{\mathrm{c}}$, \\ Franz Soplopuco Palacios ${ }^{a}$, Necemio Aranda Pretell ${ }^{a}$, Yemmy Pérez Valverde ${ }^{a}$, \\ Julio Morón Castro ${ }^{a}$ y Andrés Reyes Torres ${ }^{a}$
}

\author{
a Servicio de Cirugía Cardíaca, Instituto Nacional Cardiovascular, INCOR, EsSalud, Lima, Perú \\ ${ }^{\mathrm{b}}$ Medicina General, Universidad Nacional Mayor de San Marcos, Lima, Perú \\ c Unidad de Cardiología Nuclear, Servicio de Ayuda al Diagnóstico, Instituto Nacional Cardiovascular, INCOR, EsSalud, Lima, Perú
}

Recibido el 7 de septiembre de 2016; aceptado el 6 de diciembre de 2016

\section{PALABRAS CLAVE \\ Revascularización de miocardio; \\ Arteria mamaria \\ interna; \\ Enfermedad \\ coronaria crónica; \\ Perú}

\begin{abstract}
Resumen
Introducción: La cirugía de revascularización de miocardio (RVM) sigue siendo el gold standard en el tratamiento de la enfermedad coronaria multiarterial. Se ha demostrado que la RVM con ambas arterias mamarias internas (AMI) tiene mejor resultado de sobrevida a largo plazo.

Metodología: Investigación retrospectiva de las cirugías de RVM con AMI bilateral, realizadas en el Instituto Nacional Cardiovascular-INCOR-EsSalud entre enero de 2012 a diciembre de 2015. Los objetivos fueron determinar la mortalidad por cualquier causa y los eventos cardiovasculares mayores a 30 días de seguimiento.

Resultados: Treinta y seis pacientes fueron operados con AMI bilateral. No tuvimos mortalidad a 30 días. Los eventos cardiovasculares mayores se presentaron en el $5.56 \%$ de los pacientes (stroke $0 \%$, infarto de miocardio posquirúrgico 5.56\%, necesidad de nueva intervención coronaria $0 \%)$. La incidencia de mediastinitis y/o reconstrucción esternal fue de $0 \%$. Siete pacientes tuvieron infección superficial de la herida, no hubo diferencia significativa entre los diabéticos y los no diabéticos ( $25 \%$ vs. $16.66 \%$, OR: $3.3, p=0.88)$ o entre los pacientes con o sin sobrepeso $(19.23 \%$ vs. $20 \%$, respectivamente, OR: 0.95 , IC $95 \%, p=0.68)$ para presentar infección de herida.
\end{abstract}

Abreviaturas: AMI, arteria mamaria interna; AMII, arteria mamaria interna izquierda; AMID, arteria mamaria interna derecha; CEC, circulación extracorpórea; $C D$, coronaria derecha; DA, descendente anterior; DE, desviación estándar; Dg, diagonal; ECVM, eventos cardiovasculares mayores; HR, hazard ratio; IAM, infarto agudo de miocardio; IC, intervalo de confianza; IMC, índice de masa corporal; Mg, marginal; OR, odds ratio; RVM, revascularización de miocardio; UCI, unidad de cuidados intensivos; VSI, vena safena interna.

* Autor para correspondencia. Jr. Coronel Zegarra 417, Lima 11, Lima, Perú. Teléfono: +51-1-4111560, anexo: 4917.

Correo electrónico: jcrioso40@hotmail.com (J.C. Ríos Ortega). 
Conclusiones: La RVM con AMI bilateral es un procedimiento seguro, con bajas tasas de mortalidad y de eventos cardiovasculares mayores a corto plazo.

(C) 2016 Instituto Nacional de Cardiología Ignacio Chávez. Publicado por Masson Doyma México S.A. Este es un artículo Open Access bajo la licencia CC BY-NC-ND (http://creativecommons. org/licenses/by-nc-nd/4.0/).

\section{KEYWORDS}

Coronary artery bypass graft surgery; Bilateral internal mammary artery; Chronic coronary artery disease; Peru
Coronary artery bypass graft surgery with bilateral internal mammary artery. Short-term results

\begin{abstract}
Introduction: Coronary artery bypass graft (CABG) surgery remains the reference standard in the treatment of multivessel coronary disease. Several studies have shown that CABG with bilateral internal mammary arteries (BIMA) has better results in long-term survival.

Methodology: A retrospective study was conducted on CABG surgeries with BIMA from January 2012 to December 2015 in the National Cardiovascular Institute, INCOR, EsSalud, Peru. The objectives were to determine the mortality and major cardiovascular events at 30 days followup.

Results: Of the 36 patients subjected to CABG surgery with BIMA, the 30 -day mortality was $0 \%$, with major cardiovascular events occurring in $5.56 \%$ of patients (Stroke 0\%, postoperative myocardial infarction $5.56 \%$, need of new coronary intervention $0 \%$ ). The incidence of mediastinitis and/or sternal reconstruction was $0 \%$. Superficial wound infection was observed in 7 patients, with there being no significant difference between diabetics and non-diabetics ( $25 \%$ vs. $16.66 \%$, $\mathrm{OR}=3.3, P=.88)$, or between patients with or without overweight $(19.23 \%$ vs. $20 \%$, respectively, $\mathrm{OR}=.95 ; 95 \% \mathrm{Cl}, P=.68)$.

Conclusions: CABG surgery with BIMA is a safe procedure, with low rates of mortality and major cardiovascular events in the short-term.

(c) 2016 Instituto Nacional de Cardiología Ignacio Chávez. Published by Masson Doyma México S.A. This is an open access article under the CC BY-NC-ND license (http://creativecommons. org/licenses/by-nc-nd/4.0/).
\end{abstract}

\section{Introducción}

Desde la década de 1950 hay reportes del uso de la arteria mamaria interna izquierda (AMII) como injerto en la revascularización de miocardio. Vineberg ${ }^{1,2}$ utilizaba las AMI suturándolas a través de túneles construidos en el músculo cardiaco del ventrículo izquierdo, con la idea de llevar sangre al tejido isquémico y crear nuevos canales de irrigación. Este procedimiento dejó de ser realizado cuando Favaloro $^{3}$, en 1971, utilizó la vena safena interna (VSI), la cual suturaba directamente en la aorta ascendente y en las arterias coronarias, creando así un baipás a las obstrucciones coronarias e iniciando la era de la revascularización de miocardio (RVM). Posteriormente Loop ${ }^{4}$ publicó en 1986 un estudio demostrando que el uso de la AMII anastomosada en la arteria descendente anterior (DA) tiene mayor permeabilidad a 10 años que la VSI, lo cual se asociaba a mayores tasas de sobrevida a 10 años, menor incidencia de angina recurrente, infarto de miocardio y menores tasas de reintervención.

Actualmente, a pesar de los continuos avances de la angioplastia percutánea, la cirugía de RVM sigue siendo el gold standard de tratamiento de la enfermedad coronaria multiarterial, y la anastomosis de la AMII a la DA es el principal baipás a realizar en toda cirugía coronaria ${ }^{5-8}$. Empero, la mayoría de los pacientes no solo tienen afectación de la DA, sino que necesitan también revascularización de la arteria circunfleja y/o de la arteria coronaria derecha (CD), por lo que para «completar» dichos baipases se usa la VSI.

Debido a los buenos resultados con el uso de la AMII, muchos cirujanos empezaron a usar la AMID como opción para revascularizar los ramos marginales $(\mathrm{Mg})$ de la arteria circunfleja o para revascularizar la $C D$, estos estudios observacionales mostraron mejor evolución clínica en los pacientes en los que se usó las $2 \mathrm{AMI}^{9-13}$. Posteriormente estudios angiográficos demostraron claramente que la permeabilidad de las $2 \mathrm{AMl}$ era mucho mayor comparada con los injertos venosos, con tasas de permeabilidad del $98 \%$ a 7 días y del $95 \%$ a 2 años y a 7 años ${ }^{14-16}$.

Taggart et al. publicaron una revisión sistemática de estudios observacionales en los que se incluyó 15,962 pacientes $(11,269$ con AMI unilateral y 4,693 pacientes con AMI bilateral); las características de la población fueron ajustadas por edad, sexo, función ventricular y diabetes mellitus como antecedente para obtener grupos homogéneos. Se reportó una mayor tasa de sobrevida en los pacientes en los que se usó AMI bilateral (hazard ratio para mortalidad $=0.81$, intervalo de confianza al 95\% [IC 95\%]: 0.70-0.94).

El mismo grupo viene realizando un estudio aleatorizado de seguimiento a 10 años (ART trial Study), 1,554 pacientes han sido sometidos a revascularización con AMI unilateral y 1,548 con AMI bilateral. Los resultados a un año demostraron similares tasas de mortalidad (AMI unilateral: 2.3\%, AMII bilateral: $2.5 \%$ ), sin embargo el uso de AMI bilateral 
se asoció a un ligero incremento del $1.3 \%$ en las tasas de reconstrucción esternal por dehiscencia o por mediastinitis (AMI unilateral: $0.6 \%$, AMII bilateral: $1.9 \%$ ). Los resultados de la evolución a 10 años proveerán evidencia de calidad en relación con la sobrevida y las complicaciones ${ }^{11,17}$.

En 2014 Gijon et al. publicaron un metaanálisis de 9 estudios observacionales, 8,270 pacientes con AMI unilateral y 7,313 con AMI bilateral; con un seguimiento medio de 9 años se observó una significativa reducción de la mortalidad usando AMI bilateral (hazard ratio = 0.79, IC 95\%: 0.75-0.84) y concluyeron, inclusive, que las tasas de mayor sobrevida con el uso de AMI bilateral se observan hasta después de la segunda década de la cirugía ${ }^{18}$.

Algunos de los factores que motivaban el no uso de la AMI bilateral eran que alargaba el tiempo de cirugía y que incrementaba el riesgo de mediastinitis, lo último en especial en pacientes diabéticos. En relación con esto Dorman et al. realizaron un estudio donde compararon el uso de AMI unilateral vs AMI bilateral en pacientes diabéticos (646 no diabéticos vs 461 diabéticos), la mortalidad a 30 días fue de $2.4 \%$ vs $3.15 \%(p=0.279)$ y la tasa de infección esternal fue de $1.7 \%$ vs $3.1 \%(p=0.179)$, respectivamente. Sin embargo, en el seguimiento se evidenció que la sobrevida media de los pacientes con AMI unilateral fue de 9.8 años vs $\mathrm{AMI}$ bilateral 13.1 años $(p=0.001)$. El uso de AMI bilateral se asoció a mayor sobrevida en el análisis de regresión de Cox $(p=0.003)^{19}$.

Con la evidencia actual las guías clínicas recomiendan el uso de AMI bilateral en pacientes menores de 70 años, e inclusive en diabéticos que no tengan obesidad mórbida y con niveles de hemoglobina glucosilada $(\mathrm{HbA} 1 \mathrm{c})<7 \%{ }^{20}$.

Sin embargo, y a pesar de la evidencia, el uso de la AMI bilateral no se ha venido realizando de rutina en la cirugía de RVM en nuestro centro.

\section{Metodología}

Realizamos una investigación retrospectiva de las cirugías de RVM realizadas en el Instituto Nacional CardiovascularINCOR-EsSalud entre enero de 2012 a diciembre de 2015. Incluimos a todos los pacientes en los que se utilizó AMI bilateral, se excluyeron los pacientes que fueron sometidos a cirugía de urgencia, choque cardiogénico, reoperación y en aquellos en los que los datos de la historia clínica no eran claros o completos.

Los objetivos fueron determinar la mortalidad por cualquier causa y los eventos cardiovasculares mayores a 30 días de seguimiento. Definimos los eventos cardiovasculares mayores con 3 indicadores, infarto agudo de miocardio (IAM) postoperatorio, stroke y la necesidad de reintervención coronaria (percutánea o quirúrgica).

Todos los pacientes fueron tratados en el postoperatorio inmediato en la unidad de cuidados intensivos posquirúrgicos, donde recibieron evaluación clínica diaria, controles ecocardiográficos en las primeras 48 horas del postoperatorio, medición de enzimas cardíacas y electrocardiograma en el postoperatorio inmediato (en la primera hora del postoperatorio) y luego diariamente durante la hospitalización. Además, si era necesario los pacientes eran sometidos a otros exámenes como tomografía o angiografía.
Tabla 1 Características de la población, $\mathrm{n}=36$ pacientes

\begin{tabular}{lll}
\hline Edad(años) & 56.5 & (DE 8.9) \\
$>65$ años & 05 & $13.9 \%$ \\
Sexo & $\mathrm{N} .^{\circ}$ & $\%$ total \\
Masculino & 34 & 94.4 \\
Femenino & 02 & 05.6 \\
Antecedentes & $\mathrm{N} .^{\circ}$ & $\%$ total \\
DM 2 & 12 & 33.3 \\
HTA & 19 & 52.8 \\
Dislipidemia & 18 & 50.0 \\
IM previo & 19 & $52.8 \%$ \\
Tabaquismo & 15 & 41.7 \\
Angina inestable & 01 & 2.8 \\
IMC $\geq 25$ & 26 & 72.2 \\
IMC $\geq 30$ & 10 & 27.8 \\
Euroscore II promedio & $1.9 \%$ & $(\mathrm{DE}: 0.63)$ \\
FEVI preoperatoria & $57.4 \%$ & $(\mathrm{DE} 12.01)$ \\
FEVI $\leq 40 \%$ & 04 & $(11.1 \%)$ \\
\hline
\end{tabular}

DE: Desviación estándar; DM: diabetes mellitus; FEVI: fracción de eyección del ventrículo izquierdo; HTA: hipertensión arterial; IM: Infarto de miocardio; IMC: índice de masa corporal.

En el análisis estadístico se utilizaron medidas de tendencia central y de dispersión, así como análisis univariado para la asociación de variables (Chi cuadrado). Se usó el software STATA 10.

\section{Resultados}

\section{Características de la población}

En el periodo comprendido entre los años 2012-2015, 48 pacientes fueron sometidos a cirugía de revascularización de miocardio con AMI bilateral en el Instituto Nacional Cardiovascular (INCOR), de los cuales solo 36 cumplieron con los criterios de selección. Los pacientes de sexo masculino fueron 34 (94.44\%) mientras que los de sexo femenino solo 2 (5.56\%). La edad promedio fue de 56.5 años y 5 pacientes tenían más de 65 años (13.9\%). En cuanto a los antecedentes el $50 \%$ presentaba dislipidemia, el $41.66 \%$ tabaquismo, el $52.77 \%$ de los pacientes tenían diagnóstico de hipertensión arterial, mientras que el $33.33 \%$ tenía diabetes mellitus tipo 2. El $52.77 \%$ presentó infarto agudo de miocardio previo y un paciente $(2.77 \%)$ fue operado en el contexto de angina inestable. La fracción de eyección del ventrículo izquierdo (FEVI) promedio fue $57.47 \%$ y 4 pacientes $(11.1 \%)$ tenían $\mathrm{FEVI} \leq 40 \%$ antes de la cirugía (tabla 1 ).

\section{Características de la cirugía}

En el año 2012 solo se realizaron 3 cirugías de RVM con AMI bilateral; sin embargo la frecuencia de estas cirugías se ha incrementado en los últimos años (debido a la evidencia del uso de AMI bilateral para mejorar sobrevida), de tal manera que en el año 2015 se han realizado 18 (el 50\% de nuestra población de estudio). De las 36 cirugías 29 (80.5\%) se realizaron con circulación extracorpórea (CEC) y las restantes 
Tabla 2 Características de las cirugías, $\mathrm{n}=36$ pacientes

\begin{tabular}{lll}
\hline RVM por años & N. ${ }^{\circ}$ & $\%$ total \\
2012 & 3 & 08.3 \\
2013 & 7 & 19.4 \\
2014 & 8 & 22.2 \\
2015 & 18 & 50.0 \\
Tipo de RVM & $\mathrm{N} .^{\circ}$ & $\%$ total \\
RVM con CEC & 29 & 80.5 \\
T. CEC promedio & 96.4 min & \\
T. pinzamiento promedio & 80.4 min & \\
RVM sin CEC & 07 & 19.5 \\
Tipos de anastomosis & $\mathrm{N} .^{\circ}$ & $\%$ total \\
AMII $\rightarrow$ DA, AMID $\rightarrow$ Mg & 13 & 36.1 \\
AMII $\rightarrow$ DA, AMID $\rightarrow$ Dg & 2 & 05.6 \\
AMII $\rightarrow$ DA, AMID $\rightarrow$ CD & 3 & 08.3 \\
AMID $\rightarrow$ DA, AMII $\rightarrow$ Mg & 18 & 50.0 \\
Arteria radial & 7 & 19.4 \\
Uso de VSI & 24 & 66.67 \\
RVM arterial completa & 12 & 33.33 \\
Tiempo promedio de cirugía & 255.3 min \\
\hline AMID: arteria mamaria interna derecha; AMII: arteria mamaria \\
interna izquierda; CD: coronaria derecha; DA: arteria descen- \\
dente anterior; Dg: arteria diagonal; Mg: arteria marginal; RVM: \\
revascularización de miocardio; T. CEC: tiempo de circulación \\
extracorpórea; VSI: vena safena interna.
\end{tabular}

se realizaron sin CEC. El tiempo operatorio promedio total fue de 255.3 minutos (aproximadamente $4 \mathrm{~h}$ ). En las RVM con CEC el tiempo de CEC promedio fue de 96.4 minutos y el tiempo de pinzamiento de la aorta ascendente fue de 80.4 minutos (tabla 2).

\section{Técnica de disección y anastomosis}

Todas las AMI fueron disecadas con la técnica de «mamaria esqueletizada»; de esta forma, realizamos la RVM desinsertando la AMID, tanto de su origen en la arteria subclavia como hacia distal en su bifurcación, para luego anastomosarla a la AMII, creando así un injerto compuesto en "Y invertida», de esta forma realizamos los baipases $\mathrm{AMII} \rightarrow \mathrm{DA}$ y $\mathrm{AMID} \rightarrow \mathrm{Mg}$ en 13 pacientes (36.1\%) y $\mathrm{AMII} \rightarrow \mathrm{DA}$, AMID $\rightarrow$ diagonal en 2 pacientes $(05.6 \%)$. Por otro lado también se realizaron baipases sin desinsertar las AMI de su origen en las arterias subclavias, de esta manera realizamos los baipases AMII $\rightarrow$ DA, AMID $\rightarrow$ CD en 3 pacientes (8.3\%) y $\mathrm{AMID} \rightarrow \mathrm{DA}$, AMII $\rightarrow$ Mg en 18 (50.0\%). Para «completar» los otros vasos a revascularizar se usaron la arteria radial en 7 pacientes (19.4\%) y la VSI en 24 pacientes (66.67\%). Además, 12 pacientes (33.33\%) recibieron revascularización completa con injertos arteriales (fig. 1).

\section{Mortalidad y complicaciones (tabla 3)}

No tuvimos mortalidad hospitalaria (30 días), y en un seguimiento promedio de 14 meses todos los pacientes permanecen con vida. tabla 3

La estancia en la unidad de cuidados intensivos fue de 2.8 días (DE: 2.3 ).

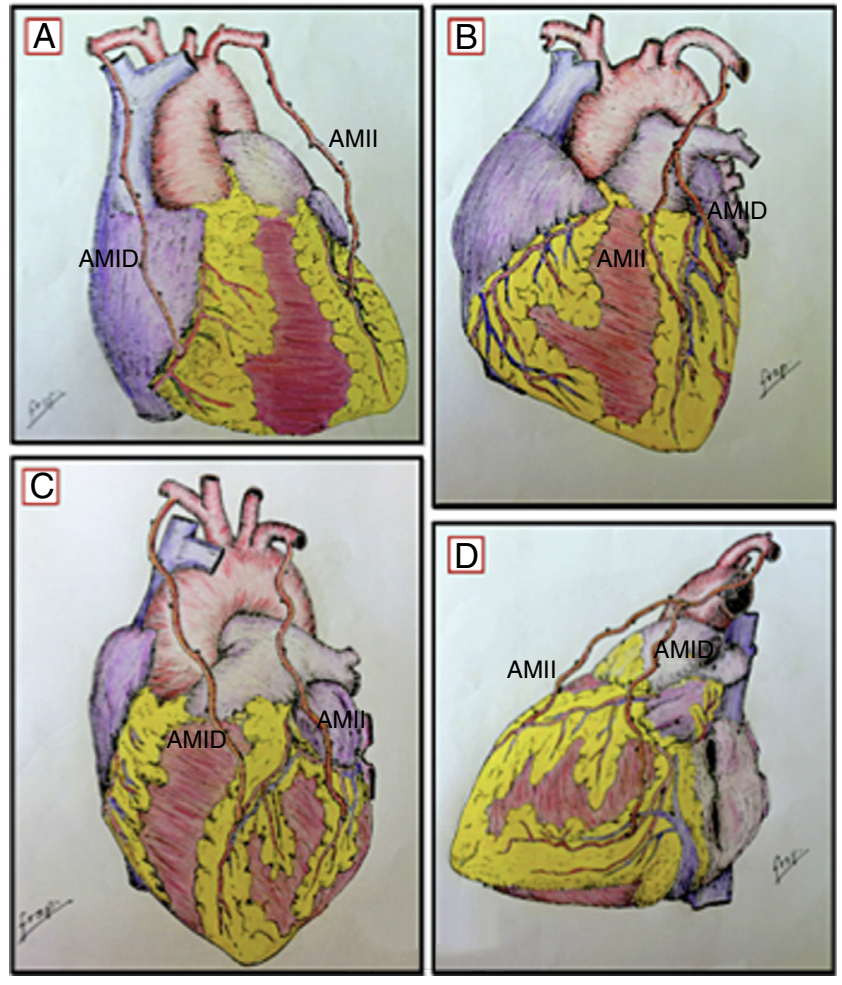

Figura 1 Técnica de anastomosis: A) $A M I I \rightarrow D A, A M I D \rightarrow C D$; B) $\quad \mathrm{AMII} \rightarrow \mathrm{DA}, \quad \mathrm{AMID} \rightarrow \mathrm{Dg} \quad$ (injerto en (Y invertida»); C) $\mathrm{AMID} \rightarrow \mathrm{DA}, \quad \mathrm{AMII} \rightarrow \mathrm{Mg}$; D) $\mathrm{AMII} \rightarrow \mathrm{DA}, \quad \mathrm{AMID} \rightarrow \mathrm{Mg}$ (injerto en «Y invertida»).AMII: arteria mamaria interna izquierda; AMID: arteria mamaria interna derecha; $C D$ : coronaria derecha; DA: descendente anterior; Dg: diagonal; Mg: marginal.

Tabla 3 Evolución postoperatoria, $\mathrm{n}=36$ pacientes

\begin{tabular}{lll}
\hline Evolución en $\mathrm{UCI}$ & $\mathrm{N} .^{\circ}$ & $\%$ total \\
Estancia promedio & 2.8 días & $\mathrm{DE} 2.3$ \\
Destete precoz VM & 06 & 16.7 \\
Complicaciones & $\mathrm{N} .^{\circ}$ & $\%$ total \\
IAM posquirúrgico & 2 & 5.5 \\
Stroke & 0 & 0.0 \\
Mediastinitis & 0 & 0.0 \\
Insuficiencia renal & 2 & 5.5 \\
Sangrado excesivo & 5 & 13.88 \\
Infección superficial de la herida & 7 & 20 \\
Mortalidad & & \\
Hospitalaria & 0 & 0.0 \\
\hline
\end{tabular}

IAM: infarto agudo de miocardio; UCI: unidad de cuidados intensivos; VM: ventilación mecánica.

Los eventos cardiovasculares mayores solo se presentaron en 2 pacientes. No hubo casos de stroke en 30 días. Sin embargo, 2 pacientes $(5.56 \%)$ presentaron criterios de infarto agudo de miocardio postoperatorio; al realizar el estudio angiográfico las AMI estaban permeables y no se necesitó angioplastia o cirugía de rescate.

Dos pacientes presentaron insuficiencia renal aguda en el postoperatorio, que requirió terapia con hemodiálisis en forma temporal. 
Infección de herida operatoria

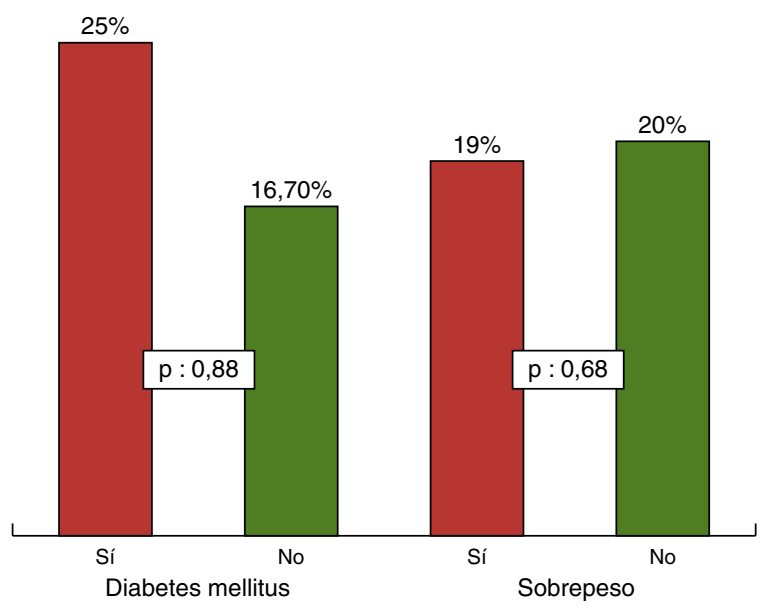

Figura 2 Infección de herida operatoria por diabetes y sobrepeso.

En las infecciones no tuvimos ningún caso de mediastinitis, ni de dehiscencia esternal que requiriera reconstrucción del mismo hueso. Sin embargo, 7 pacientes (es decir, el $19.44 \%$ del total) presentaron infección superficial de la herida operatoria con confirmación microbiológica, de los cuales 3 tuvieron dehiscencia de la misma con la consecuente reparación quirúrgica en el postoperatorio. El porcentaje de dicha infección fue similar tanto en los pacientes con sobrepeso como en aquellos con índice de masa corporal (IMC) normal (19.23\% vs. 20\%, respectivamente, OR: 0.95; IC 95\%, $p=0.68$ ). Sin embargo, se evidenció un aparente mayor porcentaje de presentación de este tipo de infección en pacientes diabéticos en comparación con pacientes no diabéticos (25\% vs. $16.66 \%$, OR: $3.3, p=0.88)$; sin embargo sin significación estadística (fig. 2).

Una de las complicaciones que se presentó con relativa frecuencia fue el sangrado postoperatorio excesivo, 5 pacientes $(13.88 \%)$ requirieron ingresar en la sala de operaciones por sangrado en el postoperatorio.

\section{Discusión}

\section{Edad}

Como se puede ver en la tabla 1 la mayoría de los pacientes fueron varones, con una edad media de 56.5 años; solo 5 pacientes tuvieron más de 65 años. Es decir, nuestra población fue una población relativamente joven y con una expectativa de vida superior a 10 años. Como hemos mencionado antes las guías clínicas actualmente recomiendan el uso de AMII bilateral en los pacientes menores de 70 años, o en aquellos con una expectativa de vida mayor a 10 años $^{20}$.

\section{Diabetes mellitus/obesidad}

La diabetes no controlada y la obesidad se asocian per se a mayor riesgo de desarrollar mediastinitis poscirugía cardiaca $^{21}$. Aunque si bien es cierto la cirugía de RVM con AMI bilateral se asocia a un ligero incremento de mediastinitis, se justifica y con creces este riesgo, porque las tasas de sobrevida son mayores cuando la RVM se realiza con AMI bilateral $^{9-11,13-19}$, y la diabetes mellitus aparentemente no incrementa este riesgo. Sin embargo, cabe mencionar que los trabajos de investigación incluyen a pacientes diabéticos controlados con niveles de $\mathrm{HbA} 1 \mathrm{c}<7 \%{ }^{19}$. En nuestra investigación incluimos 12 pacientes con antecedente de diabetes mellitus (33\% de la población), todos eran diabéticos controlados con niveles HbA1c < 7\%, y también 26 (72.2\%) pacientes tenían sobrepeso/obesidad, sin embargo ninguno de ellos presentaba obesidad mórbida $(I M C>40)$. Como se mencionó antes no se presentó ningún caso de mediastinitis o de dehiscencia esternal, de tal manera que no está contraindicado realizar cirugía de RVM con AMII bilateral en pacientes diabéticos y/u obesos, sin embargo los pacientes diabéticos deben tener niveles de glucemia y de HbA1c normales antes de la cirugía, por lo que podría ser adecuado que aquellos pacientes no controlados sean hospitalizados varios días antes de la fecha de la cirugía para mejorar sus niveles de glucemia y de HbA1c. Además, los pacientes obesos mórbidos deberían ser sometidos a un programa intensivo para disminuir en lo posible su IMC antes de la cirugía de RVM.

\section{Técnica de disección}

Se han descrito 2 técnicas de disección para las AMI, la «esqueletizada» y la pediculada; en la primera los vasos venosos mamarios se preservan íntegros e in situ, es decir, no se desinsertan ni se lesionan en su ubicación anatómica en el tórax, por el contrario en la técnica pediculada la arteria mamaria se diseca junto a los vasos venosos y con el tejido circundante. Con relación a esto Benedetto et al. ${ }^{22}$, en un estudio de 219 pacientes con mediastinitis posquirúrgica, han probado que cuando se usa $\mathrm{AMI}$ unilateral no existe diferencia en relación con el porcentaje de mediastinitis con cualquiera de las técnicas, sin embargo cuando se usa AMI bilateral la técnica de disección pediculada incrementa el riesgo de presentar mediastinitis posquirúrgica $(O R=1.80$; IC 95\%: 1.23-2.63). De igual forma, Barros Oliveira et al. ${ }^{23}$ realizaron un metaanálisis de 2,633 pacientes, demostraron que cuando se disecaban ambas AMI la técnica esqueletizada tenía menor incidencia de mediastinitis $(O R=0.327$; IC 95\%: 0.217-0.492; $p<0.001)$. Otros investigadores han encontrado resultados parecidos ${ }^{24}$. Debido a esto, en todos nuestros pacientes se realizó la técnica de disección esqueletizada y no tuvimos casos de infección esternal con necesidad de reoperación para reconstrucción ósea.

\section{Mortalidad/eventos cardiovasculares mayores}

No tuvimos mortalidad hospitalaria en nuestra serie, y los eventos cardiovasculares mayores (stroke + IAM posquirúrgico + nueva intervención coronaria) se presentaron en el $5.56 \%$ de los pacientes (2 IAM posquirúrgicos). En relación con la mortalidad otros autores reportan tasas entre $1-2 \%$ con el uso de $A M I$ bilateral, no habiendo mayores diferencias con la $\mathrm{AMI}$ unilateral ${ }^{12,17}$; sin embargo, como ya hemos mencionado, las diferencias de sobrevida se ven 
en el seguimiento a largo plazo, inclusive después de la primera década se sobrevida.

La incidencia de stroke ha sido descrita en $<3 \%{ }^{12,17}$, en ninguno de nuestros pacientes se presentó esta complicación. En relación con el IAM postoperatorio algunos estudios han descrito una incidencia de hasta el 10\% en la cirugía de RVM en general, sin embargo en estudios de RVM con AMI bilateral ha sido descrito en $<4 \%^{12,17,25}$, en nuestra serie la incidencia fue del $5.56 \%$. Lo que sí es preocupante es la alta tasa de reoperación por sangrado excesivo que se presentó en nuestros pacientes $(13.88 \%)$, ya que otros autores reportan tasas mucho más bajas, como $1.15 \%{ }^{12}$.

\section{Conclusiones}

La RVM con AMI bilateral es un procedimiento seguro, inclusive en pacientes diabéticos controlados, con bajas tasas de mortalidad y de eventos cardiovasculares mayores a corto plazo.

\section{Responsabilidades éticas}

Protección de personas y animales. Los autores declaran que para esta investigación no se han realizado experimentos en seres humanos ni en animales.

Confidencialidad de los datos. Los autores declaran que han seguido los protocolos de su centro de trabajo sobre la publicación de datos de pacientes.

Derecho a la privacidad y consentimiento informado. Los autores declaran que en este artículo no aparecen datos de pacientes.

\section{Financiación}

No se recibió patrocinio de ningún tipo para llevar a cabo este artículo.

\section{Conflicto de intereses}

Los autores declaran no tener conflicto de intereses.

\section{Bibliografía}

1. Vineberg A. Development of an anastomosis between the coronary vessels and a transplanted internal mammary artery. Can Med Assoc J. 1941;45:295-8.

2. Vineberg A. Coronary anastomosis by internal mammary artery implantation. Can Med Assoc J. 1958;78:871-4.

3. Favaloro R, Effler Cheanvechai C, Quint R, et al. Acute coronary insufficiency (impending myocardial infarction and myocardial infarction). Surgical treatment by the saphenous vein graft technique. Am J Cardiol. 1971;28:598-617.

4. Loop F, Lytle B, Cosgrove D, et al. Influence of the internal mammary artery graft on 10 years survival and others cardiac events. New Eng J Med. 1986;314:1-6.

5. Hillis LD, Smith PK, Anderson JL, et al. 2011 ACCF/AHA guideline for coronary artery bypass graft surgery: A report of the American College of Cardiology Foundation/American Heart
Association Task Force on Practice Guidelines. J Am Coll Cardiol. 2011;58:e123-210.

6. Yusuf S, Zucker D, Peduzzi P, et al. Effect of CABG surgery on survival: Overview of 10-year results from randomised trials by the CABG trialists collaboration. Lancet. 1994;344: 563-70.

7. Taggart DP, Thomas B. Ferguson Lecture. Coronary artery bypass grafting is still the best treatment for multivessel and left main disease, but patients need to know. Ann Thorac Surg. 2006;82:1966-75.

8. Serruys PW, Morice MC, Kappetein AP, et al., SYNTAX Investigators. Percutaneous coronary intervention versus coronaryartery bypass grafting for severe coronary artery disease. N Engl J Med. 2009;360:961-72.

9. Lytle BW, Blackstone EH, Loop FD, et al. Two internal thoracic artery grafts are better than one. J Thorac Cardiovasc Surg. 1999;117:855-72.

10. Buxton BF, Ruengsakulrach $P$, Fuller J, et al. The right ITA graft-benefits of grafting the left coronary system and native vessels with a high grade stenosis. Eur J Cardiothorac Surg. 2001;18:255-61.

11. Taggart DP, D'Amico R, Altman DG. The effect of arterial revascularization on survival: A systematic review of studies comparing bilateral and single internal mammary arteries. Lancet. 2001;358:870-5.

12. Ioannidis JP, Galanos O, Katritsis D, et al. Early mortality and morbidity of bilateral versus single internal thoracic artery revascularization: Propensity and risk modeling. J Am Coll Cardiol. 2001;37:521-8.

13. Rizzoli G, Schiavon L, Bellini P. Does the use of bilateral internal mammary artery (IMA) grafts provide incremental benefit relative to the use of a single IMA graft? A meta-analysis approach. Eur J Cardiothorac Surg. 2002;22:781-6.

14. Endo M, Nishida H, Tomizawa $Y$, et al. Benefit of bilateral over single internal mammary artery grafts for multiple coronary artery bypass grafting. Circulation. 2001;104: 2164-70.

15. Calafiore AM, Contini M, Vitolla G, et al. Bilateral internal thoracic artery grafting: Long-term clinical and angiographic results of in situ versus $Y$ grafts. J Thorac Cardiovasc Surg. 2000;120:990-6.

16. Dion R, Glineur D, Derouck D, et al. Long-term clinical and angiographic follow-up of sequential internal thoracic artery grafting. Eur J Cardiothorac Surg. 2000;17:407-14.

17. Taggart DP, Altman DG, Gray AM, et al. Randomized trial to compare bilateral vs. single internal mammary coronary artery bypass grafting: 1-year results of the Arterial Revascularisation Trial (ART). Eur Heart J. 2010;31:2470-81.

18. Yi G, Shine B, Rehman S, et al. Effect of bilateral internal mammary artery grafts on long-term survival. A metaanalysis approach. Circulation. 2014;130:539-45.

19. Dorman M, Kurlansky P, Traad E, et al. Bilateral internal mammary artery grafting enhances survival in diabetic patients. Circulation. 2012;126:2935-42.

20. Kolh P, Windecker S, Alfonso F, et al. Task force on myocardial revascularization of the European Society of Cardiology and the European Association for Cardio-Thoracic Surgery. European Association of Percutaneous Cardiovascular Interventions 2014 ESC/EACTS Guidelines on myocardial revascularization: The task force on myocardial revascularization of the European Society of Cardiology (ESC) and the European Association for Cardio-Thoracic Surgery (EACTS). Developed with the special contribution of the European Association of Percutaneous Cardiovascular Interventions (EAPCI). Eur J Cardiothorac Surg. 2014;46:517-92.

21. Ridderstolpe L, Gill H, Grandfeldt H, et al. Superficial and deep sternal wond complications: Incidence, risk factors and mortality. Eur J Cardiothoracic Surg. 2001;20:1168-75. 
22. Benedetto U, Altman D, Gerry S, et al. Pedicled and skeletonized single and bilateral thoracic artery grafts and the incidence of sternal wound complications: Insights from the arterial revascularization trial. J Thorac Cardiovasc Surg. 2016;152: 270-6.

23. Barros-Oliveira M, Ferraz P, de Andrade H, et al. Skeletonized versus pedicled bilateral internal mammary artery grafting: Outcomes and concerns analyzed through a meta-analytical approach. Int J Surg. 2015;16B:146-52.
24. Saso S, James D, Vecht JA, et al. Effect of skeletonization of the internal thoracic artery for coronary revascularization on the incidence of sternal wound infection. Ann Thorac Surg. 2010;89:661-70.

25. Alexander JH, Hafley G, Harrington RA, et al., for the PREVENT IV Investigators. Efficacy and safety of edifoligide, an E2F transcription factor decoy, for prevention of vein graft failure following coronary artery bypass graft surgery: PREVENT IV: A randomized controlled trial. JAMA. 2005;294:2446-54. 